\title{
The Influence of Controller Parameters on the Quality of the Train Converter Current
}

\author{
M. Brenna, F. Foiadelli, and D. Zaninelli \\ Dipartimento di Energia, Politecnico di Milano, Piazza Leonardo da Vinci 32, 20133 Milano, Italy \\ Correspondence should be addressed to F. Foiadelli, federica.foiadelli@polimi.it
}

Received 15 November 2010; Revised 25 March 2011; Accepted 31 March 2011

Academic Editor: C. M. Liaw

Copyright () 2011 M. Brenna et al. This is an open access article distributed under the Creative Commons Attribution License, which permits unrestricted use, distribution, and reproduction in any medium, provided the original work is properly cited.

This paper presents a stability analysis of train converters in order to evaluate how the controller parameters affect the absorbed current. The new dynamic model presented in this paper is capable of considering the time-variant nature of the system for the correct tuning of the feedback proportional-integral PI controller, applying a current controlled modulation technique never used in high-power traction converters. The reduction of the harmonic content of the current absorbed by a converter employed at the input stage onboard high-speed trains is really important, considering the interaction with the signaling system set up for traffic control. A computer model of the converter, considering both the power and the control structure, has also been implemented in order to deliver a validated tool for the developed theoretical analysis.

\section{Introduction}

The main purpose of public transport is to ensure, in every operational situation, the safety and regularity of the service provided by rolling stocks. To this purpose it is necessary that the harmonic disturbances generated by traction units are conformed to the EMC limits imposed by the compatibility with the signalling system set up for traffic control.

The propulsion motor drives and auxiliary services of the modern traction units are powered by microprocessor controlled bidirectional electronic converters. Therefore, there is a continuous energy exchange with the power supply system in large spectral contents, since the new traction units cannot be considered anymore as simple passive loads absorbing energy from the line.

Nowadays the input stage of the locomotives and high speed trains supplied in $\mathrm{AC}$ at $25 \mathrm{kV}, 50 \mathrm{~Hz}$ and $15 \mathrm{kV}$, $16.7 \mathrm{~Hz}$ are constituted by more four-quadrant (4Q) converters. In fact, the high power requested by the traction motor drives and the DC/DC converters for auxiliary services request a stable input DC voltage in the range of 1500 $1800 \mathrm{~V}$ to better exploit the modern IGBT switches. This DC link voltage has to be provided by $4 \mathrm{Q}$ converters that allow its stabilization and the power inversion during regenerative braking. The high power value of the input stage does not allow high switching frequencies for the 4Q converter. Consequently the absorbed current presents a high ripple value characterized by high harmonic current components that cannot be tolerated by the system. Indeed the track circuit used for signaling and communication for the traffic management and safety employs signal currents overlapped with the power ones. For this reason it is really important to control the current harmonic content in order to prevent the possible interference phenomena and to meet the requests of the new Technical Specification for the Interoperability [1]. First of all, it is necessary to perform a stability analysis of the $4 \mathrm{Q}$ converter. The paper presents a mathematical model for the single-phase four-quadrant (4Q) converter, introducing a method able to consider the time-variant nature of the system. The main goal of this algorithm is the analysis of the control parameter effects on the absorbed current in order to perform a correct tuning of the feedback PI controller. The developed method applies a particular modulation technique instead of the traditional PWM control, in order to better avail the converter characteristic, controlling directly the absorbed current and maintaining, at the same time, a constant switching frequency. Therefore, the main characteristic of the proposed algorithm is its application to a single-phase 


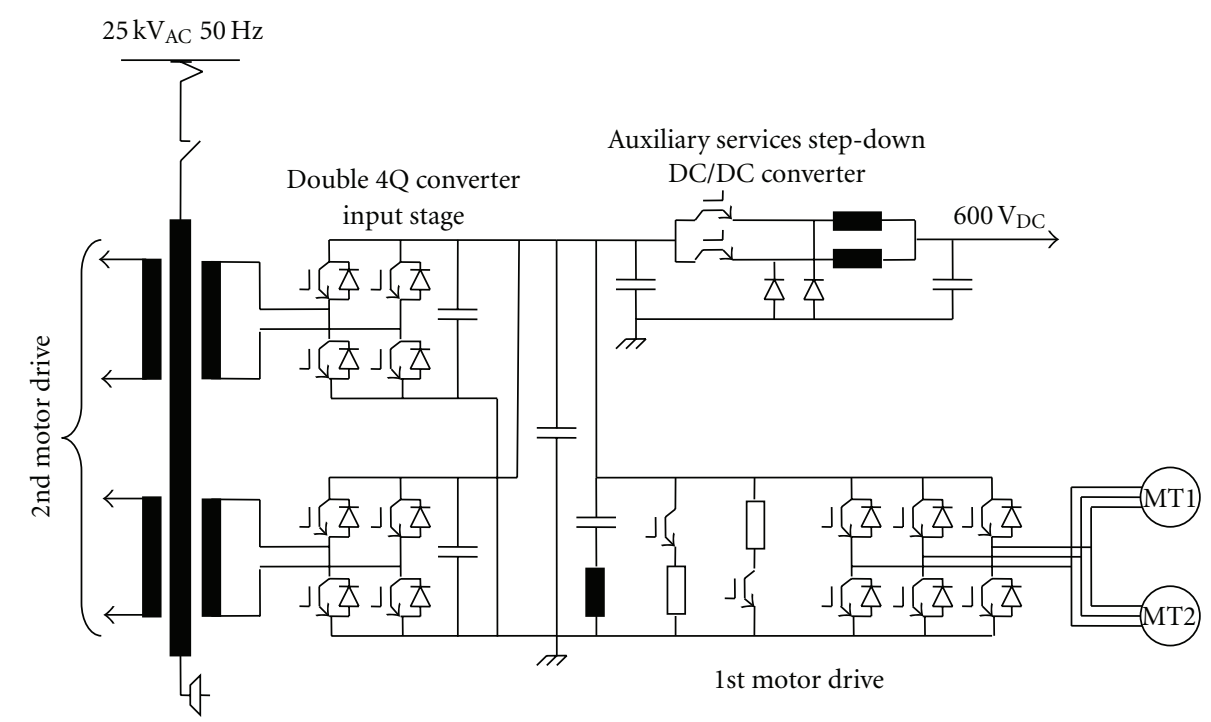

FIgURE 1: Traction circuit schematic diagram of a High Speed Train with double 4Q converters input stage.

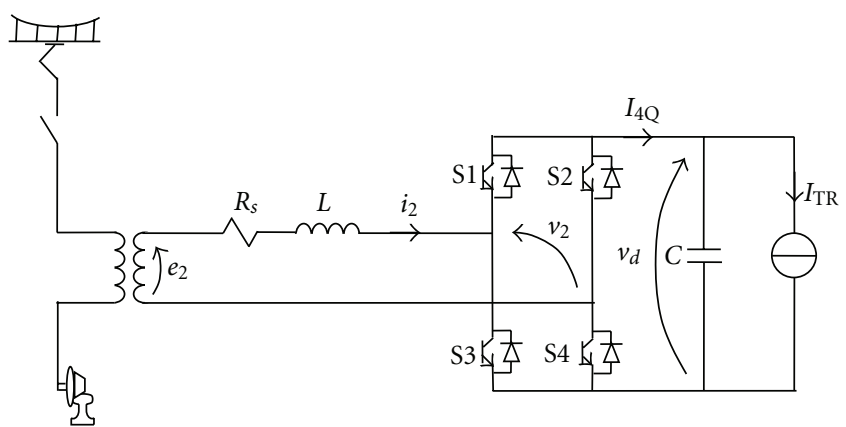

FIGURE 2: Schematic diagram of the $4 \mathrm{Q}$ converter.

converter that employs a current controlled control strategy (Smart Modulation). This allows to linearize the system in order to apply the traditional methodology for the stability analysis. Other algorithms employ vectorial techniques, Park Transform, and so forth, but they are useful for threephase converters or other methodologies are applied to the traditional PWM modulation.

Starting from real cases referred to European High-Speed Trains, a circuital model of the train input stage has been implemented in EMTP environment, considering both the power and the control structure. Many simulations have been performed to validate the theoretical analysis presented.

\section{AC/DC Conversion Input Stage}

The input stages of a High-Speed Train (HST) is composed of dedicated converters that realize the bidirectional AC/DC conversion. The HST here considered is constituted by two locomotives with two motor drives each. Every motor drive is supplied by double $4 \mathrm{Q}$ converters input stage, in order to realize redundancy and then guarantee the continuity of the service, through the main transformer as depicted in Figure 1.
The $4 \mathrm{Q}$ converter function is to absorb/deliver power from/to the AC network according to the traction motors and auxiliary services power demand [2]. First of all, the converter has to absorb current from the contact line at a voltage $e_{1}$ and frequency $f_{1}$ with a fundamental harmonic, $i_{1}$, in phase with $e_{1}$ and with limited harmonic disturbance, that means a power factor $\cos \varphi_{1}=1$ and a distortion factor $\lambda \sim 1$. Moreover, it has to absorb power from the contact line with a mean value, $P_{1}$, pulsing at the frequency $2 \cdot f_{1}$, as well as to supply a continuous power, $P_{d}$, to the three-phase motor drive inverters connected to the direct current DC link [3].

The use of a forced switching converter allows for higher DC link voltages than those generated by diode or thyristor bridges. In this manner, it is possible to increase the flowing power at the same converter losses [4]. The maximum value of the DC link voltage is imposed by the presence of the switches and the voltage smoothing capacitors [5]. Moreover, the filtering on the AC side is simplified and is only related to the switching frequencies [6].

\section{Mathematical Model of the $4 \mathrm{Q}$ Converter}

The $4 \mathrm{Q}$ converters used in modern locomotives are twolevel converters, because they employ the new high voltage IGBT. Therefore, their stability analysis needs to consider the four different circuit configurations as a function of the switch status. Figure 2 reports a representation of the two converter levels considered in which its equivalent circuit is composed of an ideal voltage generator $\left(e_{2}=e_{1} / h\right.$, where $h$ is the transformer ratio of the onboard transformer), a commutation inductance $L$, an equivalent resistances $R_{s}$, a DC link capacitance $C$, and an equivalent current absorbed by the train motor drives $I_{\mathrm{TR}}$.

The modeling process starts by representing the discrete modes of the converter mathematically. The discrete model of the converter describes each status of the converter by 
TABLE 1: State equations of a 4Q converter.

\begin{tabular}{lccc}
\hline & State variable $i_{2}$ & State variable $v_{d}$ & Matrix format \\
\hline First configuration S1, S4 ON; S2, S3 OFF & $L\left(d i_{2} / d t\right)=-R_{s} i_{2}-v_{d}+E_{2} \sin (\omega t)$ & $C\left(d v_{d} / d t\right)=i_{2}-I_{\mathrm{TR}}$ & $A \cdot \dot{x}=B \cdot x+K$ \\
Second configuration S1, S4 OFF; S2, S3 ON & $L\left(d i_{2} / d t\right)=-R_{s} i_{2}+v_{d}+E_{2} \sin (\omega t)$ & $C\left(d v_{d} / d t\right)=-i_{2}-I_{\mathrm{TR}}$ & $A \cdot \dot{x}=B^{\prime} \cdot x+K$ \\
Third configuration S1, S2 ON; S3, S4 OFF & $L\left(d i_{2} / d t\right)=-R_{s} i_{2}+E_{2} \sin (\omega t)$ & $C\left(d v_{d} / d t\right)=-I_{\mathrm{TR}}$ & $A \cdot \dot{x}=B^{\prime \prime} \cdot x+K$ \\
Fourth configuration S1, S2 OFF; S3, S4 ON & $L\left(d i_{2} / d t\right)=-R_{s} i_{2}+E_{2} \sin (\omega t)$ & $C\left(d v_{d} / d t\right)=-I_{\mathrm{TR}}$ & $A \cdot \dot{x}=B^{\prime \prime} \cdot x+K$ \\
\hline
\end{tabular}

$\omega$ is the supply voltage angular frequency, $x=\left[\begin{array}{ll}i_{2} & v_{d}\end{array}\right]^{T}$ is the state vector, $\dot{x}$ is its time derivative, $A$ contains the conservative elements of the converter, $B$, $B^{\prime}$, and $B^{\prime \prime}$ are the matrices of the state variables coefficients that represent the specific working conditions, and $K$ is the forcing term vector. The switching operation of the $4 \mathrm{Q}$ converters realizes three different state conditions (because the third and fourth configurations bring to the same circuit topology) characterized by different matrices $B, B^{\prime}$, and $B^{\prime \prime}$.

separate linear equations. Taking the state variables as $i_{2}$ and $v_{d}$, the four-converter state equations are reported in Table 1.

Observing the matrices $B, B^{\prime}$, and $B^{\prime \prime}$, it is possible to note that the working conditions do not influence the elements on the diagonal. Therefore, the state equation in generic condition can be reduced to

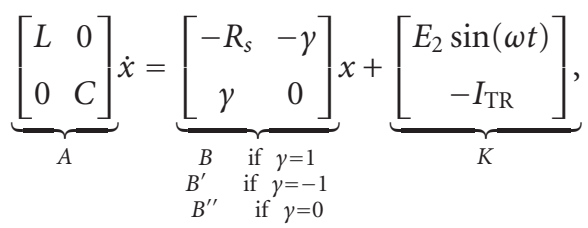

where $\gamma$ depends on the mode of the converter and can be equal to $1,-1$, or 0 .

The $4 \mathrm{Q}$ converter function is to absorb or deliver power from/to the AC line depending on the power value requested by the traction motors and auxiliary services. This objective can be achieved using different control logic [7]. For this application PWM is often used, which is a well-known commutation technique. This control has the advantage of having a constant switching frequency, which allows for the sizing of dedicated filters tuned at that frequency; however, this control has a disadvantage in that it has indirect control of the current through the variation of the modulation index, $m$, and the load angle, $\delta$, causing possible transient overcurrents.

Assuming the use of PWM modulation, there are many commutations in a period at the main frequency, and, therefore, $\gamma$ can be replaced with the time-varying function, $\gamma=m \cdot \sin (\omega t-\delta)$, where $m$ is the modulation index and $\delta$ is the load angle. The time-varying nature of $\gamma$ complicates the resolution of (1).

To eliminate the time variance in the equations, instead of transforming the reference frame and applying the Park Transform, a substitution method has been adopted [8]. The first step is to rewrite the state equations including $\gamma$ :

$$
\begin{gathered}
L \frac{d i_{2}}{d t}+R_{s} i_{2}=-m \sin (\omega t-\delta) v_{d}+E_{2} \sin (\omega t), \\
C \frac{d v_{d}}{d t}=m \sin (\omega t-\delta) i_{2}-I_{\mathrm{TR}} .
\end{gathered}
$$

From (2) a solution for $i_{2}$ and $v_{d}$ can be

$$
\begin{gathered}
i_{2}=i \sin (\omega t+\varphi)+I_{20}, \\
v_{d}=v_{0}+v_{r} \cos (2 \omega t+\vartheta),
\end{gathered}
$$

where $i_{2}$ has one constant forcing term and another sinusoidal term at the primary angular frequency, $\omega$, and $v_{d}$ has one constant component $v_{0}$ and a ripple at an angular frequency of $2 \omega$. Both the constant term $I_{20}$ and phase $\varphi$ are forced to zero by the converter control. The ripple component, $v_{r}$, is quite low due to an LC filter in the DC link that is tuned at a frequency, $f_{2}=2 \cdot f_{1}$, as well as a high capacitance and voltage in the DC link. As it will be presented in the next session, it reaches the $0.2 \%$ of the DC component, therefore, the voltage $v_{d}$ can be approximated with its average value $v_{0}$.

Deriving (3) and substituting it into (2), a new system of three equations in three unknown terms $\left(v_{0}, m, \delta\right)$ can be obtained equating the cosine and sine terms:

$$
\begin{gathered}
L \frac{d i}{d t}+R_{s} i=E_{2}-m v_{0} \cos \delta, \\
L i \omega=m v_{0} \sin (\delta), \\
C \frac{d v_{0}}{d t}=\frac{1}{2} m i \cos (-\delta)-I_{\mathrm{TR}} .
\end{gathered}
$$

These equations are time invariant, but nonlinear. To be implemented in a control system design they must be linearized around their steady-state operating point. To linearize the system, the following substitutions are made, where capital letters represent the steady-state operating point and the prime symbol represents a small deviation from the operating point:

(i) $m=M+m^{\prime}$ modulation index;

(ii) $v_{0}=V_{0}+v_{0}^{\prime}$ DC link voltage;

(iii) $\delta=\Delta+\delta^{\prime}$ load angle;

(iv) $i=I+i^{\prime}$ absorbed current.

The value of $E_{2}$ is stiff and imposed by the contact line connected to the main line, whereas $I_{\mathrm{TR}}$ is imposed by the power requested by the train.

The first-order approximations from the Taylor Series for the sine and cosine functions are $\sin (X+x) \approx \sin X+x \cos X$ 
and $\cos (X+x) \approx \cos X-x \sin X$ used to simplify the system of (4):

$$
\begin{aligned}
L \frac{d i^{\prime}}{d t}+R_{s}\left(I+i^{\prime}\right)= & E_{2}-\left(M+m^{\prime}\right)\left(V_{0}+v_{0}^{\prime}\right) \cos \Delta \\
& +\left(M+m^{\prime}\right)\left(V_{0}+v_{0}^{\prime}\right) \delta^{\prime} \sin \Delta, \\
L\left(I+i^{\prime}\right) \omega= & \left(M+m^{\prime}\right)\left(V_{0}+v_{0}^{\prime}\right) \sin \Delta \\
& +\left(M+m^{\prime}\right)\left(V_{0}+v_{0}^{\prime}\right) \delta^{\prime} \cos \Delta, \\
C \frac{d v_{0}^{\prime}}{d t}= & \frac{1}{2}\left(M+m^{\prime}\right)\left(I+i^{\prime}\right) \cos (-\Delta) \\
& -\frac{1}{2}\left(M+m^{\prime}\right)\left(I+i^{\prime}\right) \delta^{\prime} \sin \Delta-I_{\mathrm{TR}} .
\end{aligned}
$$

\section{Stability Analysis of $4 Q$ Converter with Current Controlled Strategy}

Retaining only the first-order terms, it is possible to obtain the small-signal model, useful to find the frequency response of the converter [9]:

$$
\begin{gathered}
L \frac{d i^{\prime}}{d t}+R_{s} i^{\prime}=-M v_{0}^{\prime} \cos \Delta-m^{\prime} V_{0} \cos \Delta, \\
L i^{\prime} \omega=M v_{0}^{\prime} \sin \Delta+m^{\prime} V_{0} \sin \Delta, \\
C \frac{d v_{0}^{\prime}}{d t}=\frac{1}{2} M i^{\prime} \cos (-\Delta)+\frac{1}{2} m^{\prime} I \cos (-\Delta) .
\end{gathered}
$$

The small-signal model is developed in terms of the Laplace operator. Applying the Laplace transformation to (6) and assuming zero initial conditions, we obtain

$$
\begin{gathered}
\left(L s+R_{s}\right) i^{\prime}=-M v_{0}^{\prime} \cos \Delta-m^{\prime} V_{0} \cos \Delta, \\
L i^{\prime} \omega=M v_{0}^{\prime} \sin \Delta+m^{\prime} V_{0} \sin \Delta, \\
C s v_{0}^{\prime}=\frac{1}{2} M i^{\prime} \cos (-\Delta)+\frac{1}{2} m^{\prime} I \cos (-\Delta) .
\end{gathered}
$$

Instead of the PWM control that generates an AC voltage trough the modulation index $m$ and its phase $\delta$, in the following a new modulation technique that can directly control the current value is proposed.

Considering the variability of the switching frequency, $f_{s}$, and the difficulty defining an adequate current ripple, the Smart Modulation, presented in $[10,11]$ and briefly described in the appendix, is applied.

This strategy allows direct control of the current value maintaining, at the same time, a constant value of the switching frequency. Therefore, the modulation index, $m$, does not directly appear in the control algorithm, but, assuming a unitary power factor, the converter absorbed current is controlled by the equivalent conductance, G. From the second equation of the system in (7) it is possible to determine the value of $m^{\prime}$. Substituting $m^{\prime}$ into the third equation of (7) we obtain

$$
\begin{aligned}
s C v_{0}^{\prime}= & \frac{1}{2} M i^{\prime} \cos (-\Delta)-\frac{1}{2} I \cos (-\Delta) \frac{s L+R_{s}}{V_{0} \cos \Delta} i^{\prime} \\
& -\frac{1}{2} I \cos (-\Delta) \frac{M}{V_{0}} v_{0}^{\prime}, \\
v_{0}^{\prime}= & \frac{\left((1 / 2) M \cos (\Delta)-(1 / 2) I\left(\left(s L+R_{s}\right) / V_{0}\right)\right)}{\left(s C+(1 / 2) I \cos (\Delta)\left(M / V_{0}\right)\right)} i^{\prime} .
\end{aligned}
$$

Equation (8) gives the value, $v_{0}^{\prime}$, as a function of $i^{\prime}$. It is now possible to determine the variations of the DC link voltage for small changes of the current absorbed by the converter

$$
\begin{aligned}
\frac{\partial v_{0}^{\prime}}{\partial i^{\prime}} & =\frac{\left((1 / 2) M \cos (\Delta)-(1 / 2) I\left(\left(s L+R_{s}\right) / V_{0}\right)\right)}{\left(s C+(1 / 2) I \cos (\Delta)\left(M / V_{0}\right)\right)} \\
& =\frac{-\left((1 / 2) L\left(I / V_{0}\right)\right) s+(1 / 2) M \cos (\Delta)-(1 / 2) R_{s}\left(I / V_{0}\right)}{(C) s+(1 / 2) I \cos (\Delta)\left(M / V_{0}\right)} .
\end{aligned}
$$

The reference current is given by the product between the equivalent conductance, $G$, and the supply voltage, $E_{2}(i=$ $G \cdot E_{2}$ ), where $\mathrm{G}$ comes from the PI regulator that maintains the DC link voltage constant:

$$
G=\left(k_{p}+\frac{k_{i}}{s}\right)\left(V_{0 \mathrm{ref}}-V_{0}\right) .
$$

The block diagram of the DC voltage regulation loop is shown in Figure 3, where only one of the two-input stage $4 \mathrm{Q}$ converter is represented. In the block diagram shown in Figure 3 three control loops can be identified. The first control loop is the DC link voltage control loop comprised of the voltage measurement, the low-pass filter explained above, the comparison with the reference value, $V_{0 \text { ref, }}$, and the PI controller. Its output is the value of the equivalent conductance, $G$, that keeps the DC link voltage constant while varying the power requested or injected by the traction motors and auxiliary services. The second loop is the reference current generator, which considers the input voltage measurement followed by a filter dedicated to high-frequency disturbances. The obtained value from this loop multiplied with the equivalent conductance, $G$, gives the reference current that the converter, through Smart Modulation, has to generate in order to balance the input and output powers. The third loop is related to the DC component compensation in the AC input current. In fact, its output value is a constant current that, when added to the reference current, allows the DC component to be cancelled out in order to avoid saturation of the input transformer.

The DC link voltage variation for small changes of $G$ given by the regulator is obtained multiplying (9) for the input voltage $E_{2}$ :

$$
\frac{\partial v_{0}^{\prime}}{\partial G}=\frac{\partial v_{0}^{\prime}}{\partial i^{\prime}} \cdot E_{2} .
$$


TABle 2: Main data of the considered High Speed Train input stage converter.

\begin{tabular}{lcc}
\hline & Value & M.U. \\
\hline$E_{2 \mathrm{MAX}}$ & 1000 & $\mathrm{~V}$ \\
$V_{0}$ & 1800 & $\mathrm{~V}$ \\
$P_{\text {ass }}$ & 900 & $\mathrm{~kW}$ \\
$I_{\mathrm{TR}}$ & 500 & $\mathrm{~A}$ \\
$I_{\mathrm{MAX}}$ & 1800 & $\mathrm{~A}$ \\
$f_{\text {sw }}$ & 500 & $\mathrm{~Hz}$ \\
\hline
\end{tabular}

The open-loop function given by the product of (10) and (11) is

$F T_{\mathrm{OL}}$

$=\left(k_{p}+\frac{k_{i}}{s}\right) \cdot \frac{\partial v_{0}^{\prime}}{\partial G}$

$=\left(k_{p}+\frac{k_{i}}{s}\right)$

$\frac{-\left((1 / 2) L I\left(E_{2} / V_{0}\right)\right) s+(1 / 2) M E_{2} \cos (\Delta)-(1 / 2) R_{s} I\left(E_{2} / V_{0}\right)}{(C) s+(1 / 2) I \cos (\Delta)\left(M / V_{0}\right)}$.

The above presented theory leads to a procedure for the converter design. In particular, the following steps can be identified:

(1) converter parameters identification, such as rated and maximum powers, AC input and DC output voltages, and consequently input and output currents;

(2) switching inductance $L$ and DC link capacitance $C$ calculation. These two parameters are determined considering the maximum input current ripple and the maximum acceptable DC link voltage oscillation;

(3) calculation of the correct tuning parameters of the regulator (proportional $k_{p}$ and the integral $k_{i}$ ) to have good stability and at the same time a fast time response of the system;

(4) calculation of the tuning parameters of the regulator (proportional $k_{p}$ and the integral $k_{i}$ ) at the limit of stability in order to perform the sensitivity analysis;

(5) sensitivity analysis.

\section{Analysis Application in a Real Case}

The converter design steps previously formulated have been applied to a real case as example consisting in the converter studied in this paper (Figure 1) employed as input stage onboard High-Speed Railway (HSR) Trains operating on the new Italian HSR lines.

Converter Parameters Identification. In transportation systems many typologies of power converters are employed that can have different applications (i.e., front-end converters, auxiliary services, UPS, high-speed trains, heavy traction, naval propulsion) and sizes. The typical size of the converter and its usual data for this application are reported in Table 2.
Switching Inductance L and DC Link Capacitance C Calculation. Referring to Figure 2, the elements that more influence the control parameters are the switching inductance $L$ and the DC link capacitance $C$. The inductance value is chosen to limit the input current ripple, while the capacitance $C$ influences the DC link oscillation. Therefore, the obtained values are $L=1.5 \mathrm{mH}$ and $C=200 \mathrm{mF}$.

Calculation of the Correct Tuning Parameters of the Regulator (Proportional $k_{p}$ and the Integral $k_{i}$ ). In order to study the system stability using the Bode criteria through module and phase diagrams, identified by the open loop transfer function (12), a dedicated code in MATLAB environment has been implemented. From this program it is possible to calculate the control parameters $k_{p}$ and $k_{i}$, giving as input the specific data of the converter considered (Table 2), that have to stabilize the converter dynamics. Under these conditions and referring to the studied case, a good compromise to obtain a fast system response and, at the same time, a good phase margin is $k_{p}=0.1$ and $k_{i}=3$. The Bode diagrams obtained from (12) using these values are shown in Figure 4(a). The cut-off frequency that guarantees a good response with a sufficient phase margin is about $200 \mathrm{~Hz}$.

Calculation of the Tuning Parameters of the Regulator (Proportional $k_{p}$ and the Integral $k_{i}$ ) at the Limit of Stability. The parameter values for the limit of the stability condition have been investigated using the same Matlab code, obtaining $k_{p}=0.2$ and $k_{i}=3$. The relative Bode diagrams for these parameters are shown in Figure 4(b). As can be seen from this figure, the phase margin is close to zero.

Sensitivity Analysis. Applying the same algorithm, it is possible to obtain the effect of changes of the parameters on the stability of the controller. As it can be shown in Figure 5, an increase of the proportional gain causes significant reduction of the gain margin leading to the loss of stability. On the contrary, the reduction of $k_{p}$ improves the stability but it degrades the time response performance. Once determined the power system structure, the traction power converters are mainly subjected to the line voltage variation. In fact, in traction systems the voltage can vary in the range of $-33 \%-+20 \%$ of the nominal one. As it can be seen in (12), the line voltage $E_{2}$ (reported on the secondary side of the onboard transformer) is common to all the numerator terms, therefore, it can be considered as a proportional gain. The sensibility analysis carried out and presented in Figure 5 shows that around the optimal value of $k_{p}(-33 \%-+20 \%)$ there is enough gain margin to maintain the stability.

\section{Harmonic Absorption due to Single-Phase Supply}

One of the problems that characterize the $4 \mathrm{Q}$ converter and, in general, all of the AC/DC single-phase converters, is the absorption of variable instantaneous power. In fact, in contrast to a balanced three-phase system, the flowing power in a single-phase system is characterized by a mean 


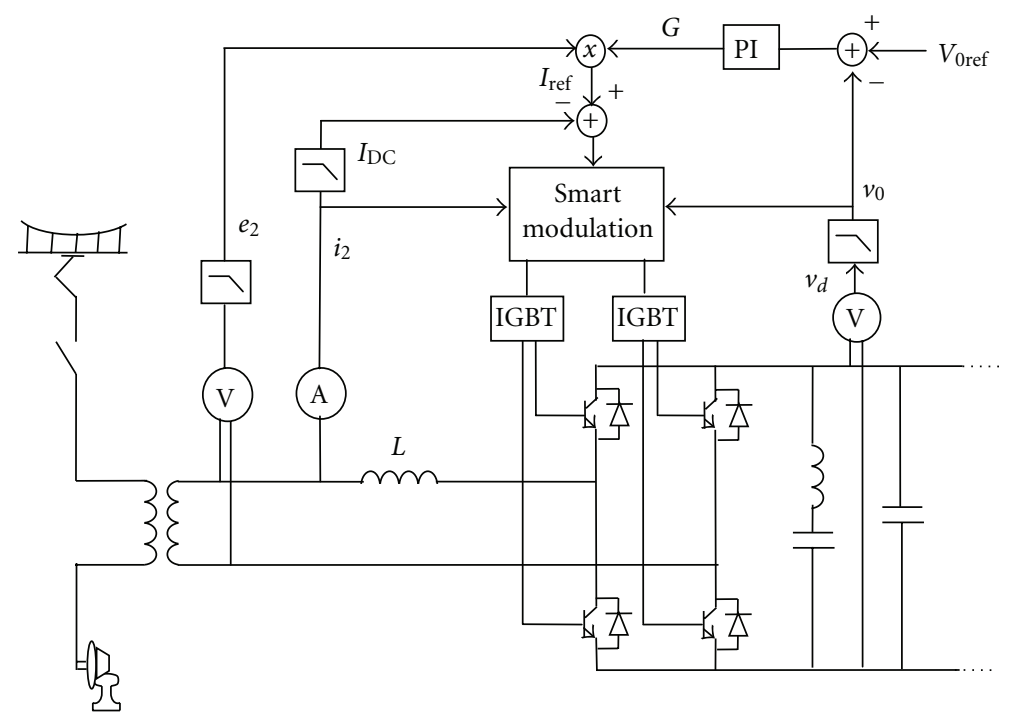

FIGURE 3: Block diagram of the system and control loops.

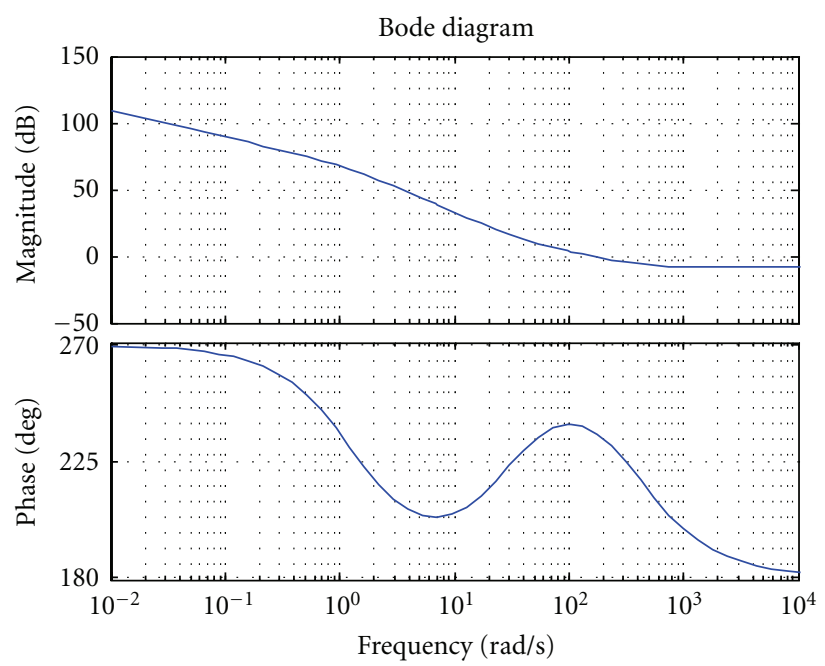

(a)

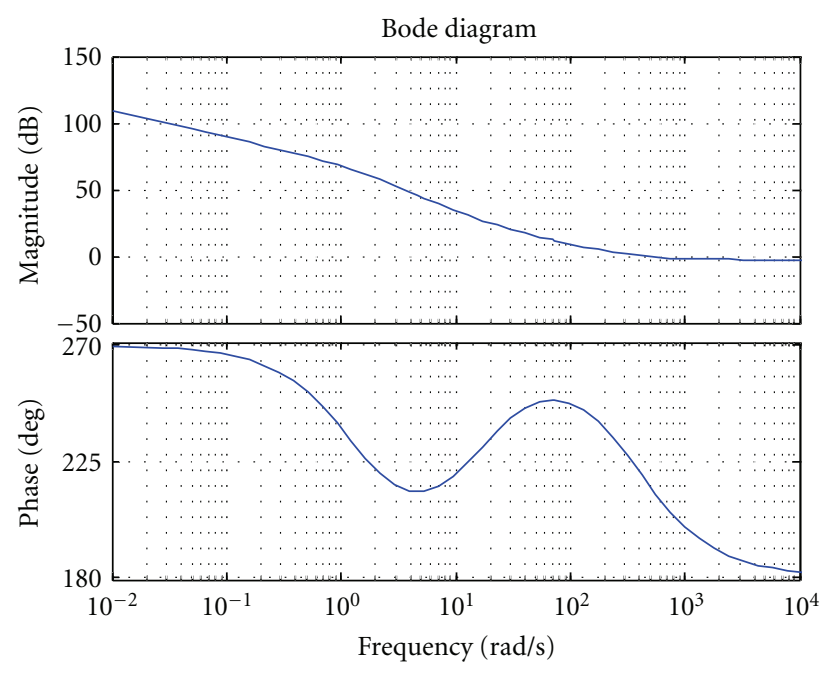

(b)

FIGURE 4: Bode diagram of the converter and regulator system for (a) $k_{p}=0.1$ and $k_{i}=3$ and (b) $k_{p}=0.2$ and $k_{i}=3$.

value equal to the active power requested from the load and a variable term oscillating at a frequency twice that of the supply frequency. This oscillation, in the case of traction converters, causes the generation of a ripple in the DC output voltage. The $4 \mathrm{Q}$ converters are equipped with a filter that is tuned to a frequency of $100 \mathrm{~Hz}$ in $25 \mathrm{kV} / 50 \mathrm{~Hz}$ train systems or $33.3 \mathrm{~Hz}$ in $15 \mathrm{kV} / 16.7 \mathrm{~Hz}$ train systems. However, it is not possible to completely cancel the ripple in the DC link voltage. Due to the regulator response time, the equivalent conductance at the end of the transient state condition coming out from the regulator can be defined as the sum of its mean theoretic value requested $G_{0}$ and a ripple at double frequency $G_{100}\left(G=G_{0}+G_{100} \sin (2 \omega t)\right)$. This phenomenon has not been considered in the stability analysis because it is not influential; however, it is the cause of harmonic current absorption from the converter. The reference current leaving the regulator, given as the product between $G$ and the sinusoidal input voltage $e_{2}=E_{2} \sin (\omega t)$, is

$$
\begin{aligned}
I_{\text {ref }} & =G \cdot e_{2}=\left[G_{0}+G_{100} \sin (2 \omega t)\right] \cdot E_{2} \sin (\omega t) \\
& =E_{2} G_{0} \sin (\omega t)+\frac{1}{2} G_{100} E_{2} \cos (\omega t)-\frac{1}{2} G_{100} E_{2} \cos (3 \omega t) .
\end{aligned}
$$

The reference current reported in (13) can be defined as the sum of the following three terms:

(i) $I_{\text {ref } 0}=E_{2} G_{0} \sin (\omega t)$ is the theoretical value of the reference current proportional to the absorption power; it is in phase with the voltage signal and at the same frequency; 


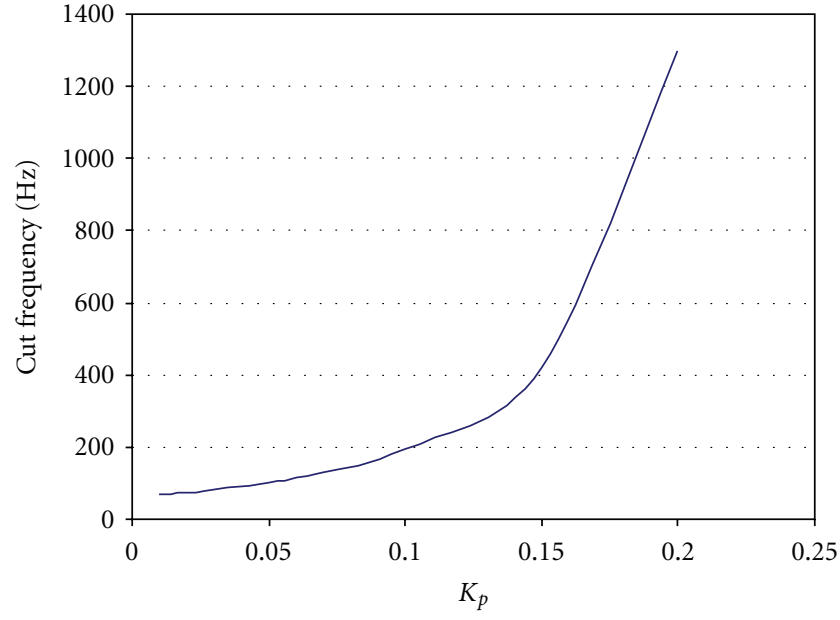

(a)

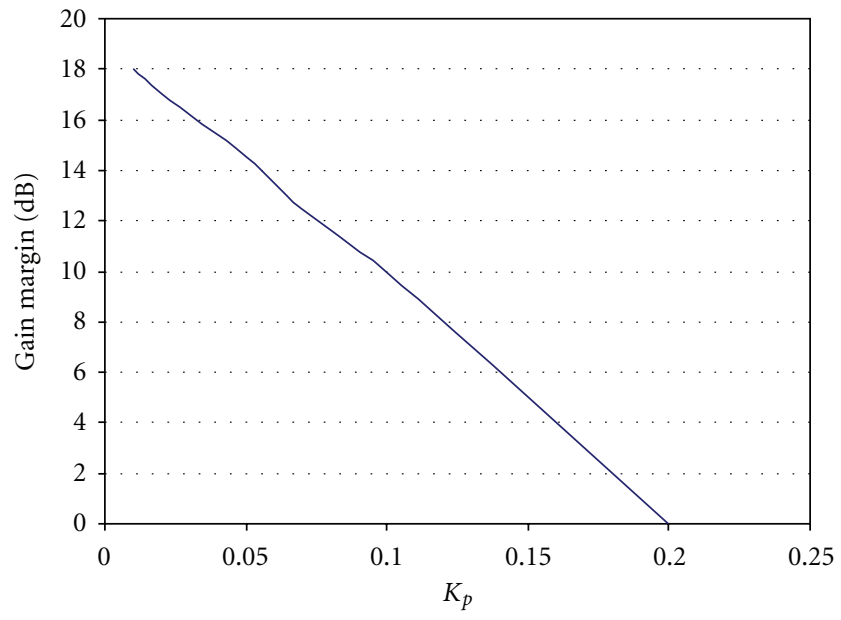

(b)

Figure 5: Influence of $k_{p}$ to the cut frequency and to the gain margin.

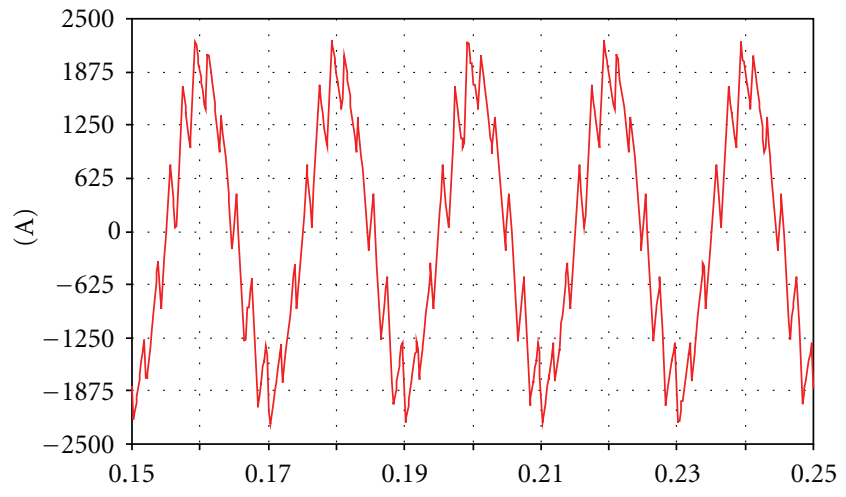

(s)

Figure 6: Current absorbed by one 4Q converter in the traction phase in stable condition $\left(k_{p}=0.1, k_{i}=3\right)$.

(ii) $I_{\operatorname{ref} \varphi}=(1 / 2) G_{100} E_{2} \cos (\omega t)$ is a term at the same net frequency, but shifted $\pi / 2$ radians, which is the source of absorption of the reactive power;

(iii) $I_{\text {ref } 3}=-(1 / 2) G_{100} E_{2} \cos (3 \omega t)$ is a term at three times the frequency that determines the presence of a third harmonic current.

In order to reduce the $G_{100}$ component, it is necessary to filter the DC link voltage with a suitable low-pass filter. This filter tuning is critical because it simultaneously allows for a fast system response and a low cut-off frequency. It is impossible to achieve both of these objectives, but for the system stability it is necessary to focus on the system response, which implies a small ripple.

\section{Model and Simulation Results}

In order to validate the theoretical analysis described above, a suitable model of the system has been implemented using the EMTP-ATP dynamic simulation tool. The data employed

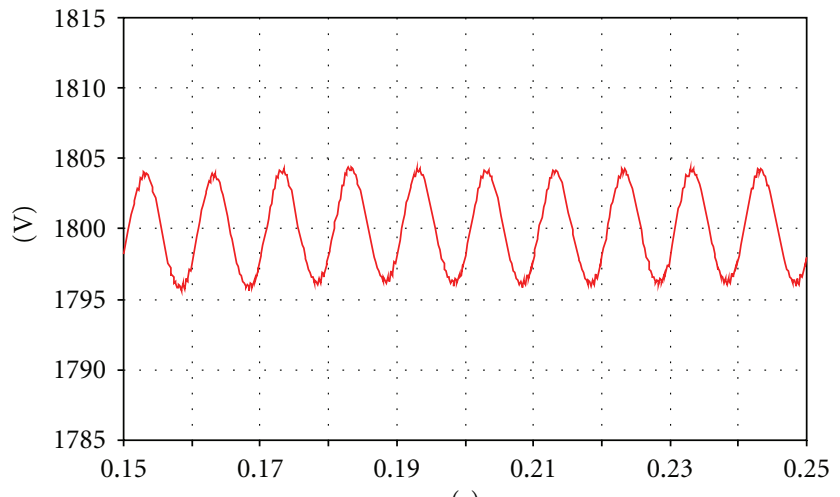

(s)

Figure 7: DC link voltage in end of transient state and stable condition $\left(k_{p}=0.1, k_{i}=3\right)$.

for the model is obtained from a real HSR Train operating in Italy on a $25 \mathrm{kV} / 50 \mathrm{~Hz}$ line. Each $4 \mathrm{Q}$ converter is sized for a rated power equal to $900 \mathrm{~kW}$ and a maximum peak power equal to $1500 \mathrm{~kW}$. These are relevant power values for a switching converter that has to be small and light enough to be installed onboard.

The $4 \mathrm{Q}$ converters consist of a power circuit and a control system. The power circuit has been modeled with the circuital elements already available in EMTP-ATP. The MODELS language has been used for implementing the proposed converter control.

In the following, some simulation results are reported in order to verify the outcomes of the performed analysis. The simulation cases considered for the theoretical analysis validation are the following:
(A) nominal condition;
(B) loading;
(C) converters regulators working at the stability limit. 


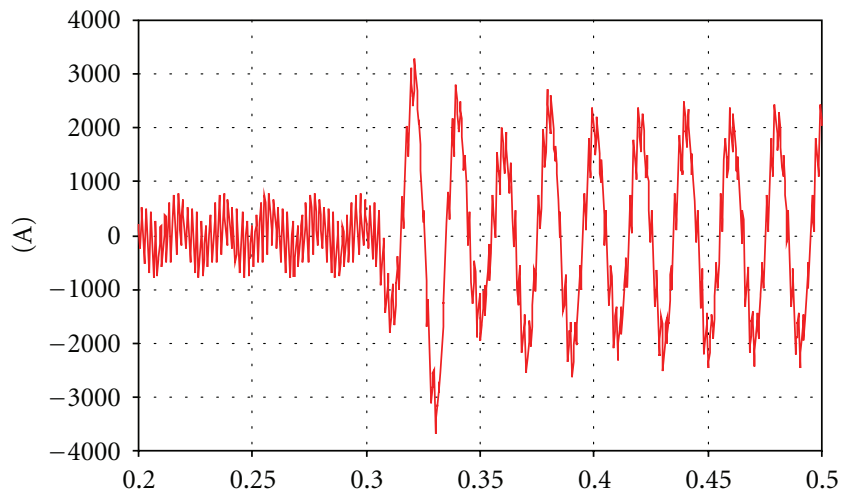

(s)

Figure 8: Loading transient of the current absorbed by one $4 \mathrm{Q}$ converter in stable condition $\left(k_{p}=0.1, k_{i}=3\right)$.

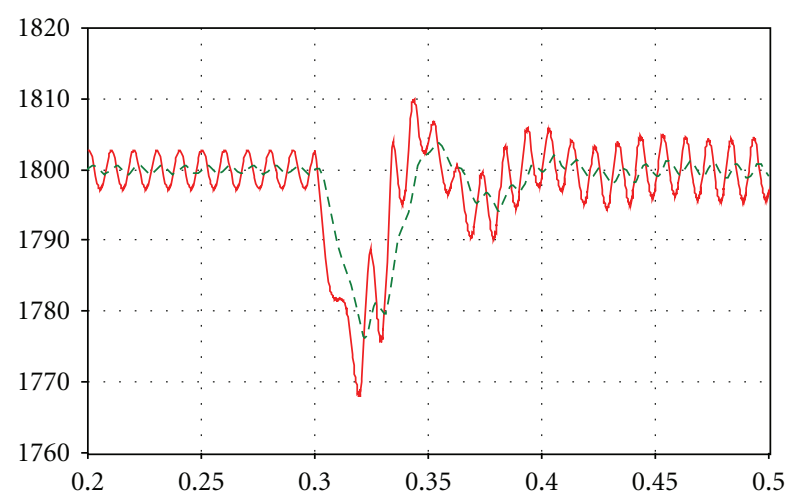

(s)

FIGURE 9: DC link voltage and filtered signal in loading phase for $k_{p}=0.1$ and $k_{i}=3$.

TABLE 3: Most significant current harmonic components in normal operating condition.

\begin{tabular}{lcc}
\hline Harm. & Amplitude $[\mathrm{A}]$ & $\mathrm{pu}$ \\
\hline 1 & 1997.5 & 1 \\
3 & 53.6 & 0.026 \\
8 & 79.3 & 0.039 \\
10 & 347.6 & 0.174 \\
12 & 68.8 & 0.034 \\
19 & 71.9 & 0.036 \\
21 & 55.9 & 0.028 \\
23 & 20.0 & 0.010 \\
28 & 31.595 & 0.016 \\
\hline
\end{tabular}

(A) Nominal Condition. The normal operating condition considers the traction phase at the nominal power of $3.6 \mathrm{MW}$ due to four $4 \mathrm{Q}$ converters. The parameters $k_{p}=0.1$ and $k_{i}=3$ obtained for the stability condition are employed in the model. The current absorbed by one $4 \mathrm{Q}$ converter is shown in Figure 6.

From Figure 6 it is easy to see that the converter commutations are numerically limited because of the low

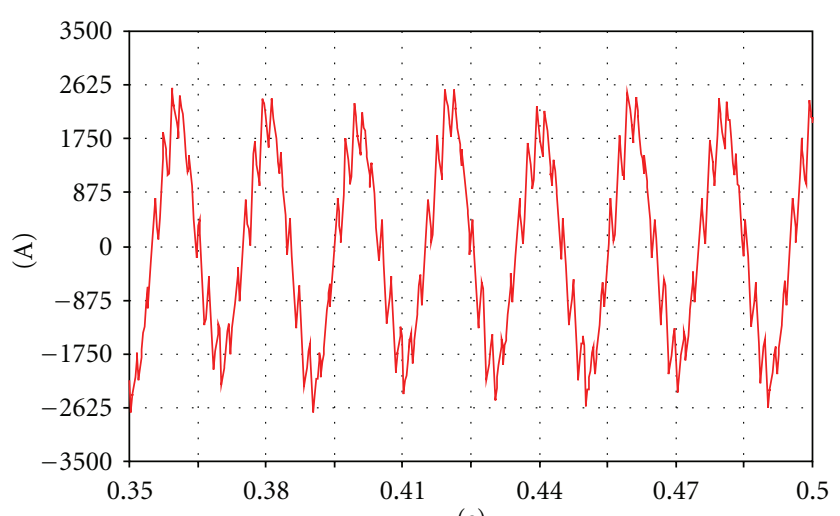

(s)

FIgURE 10: DC link current at full load for $k_{p}=0.2$ and $k_{i}=3$.

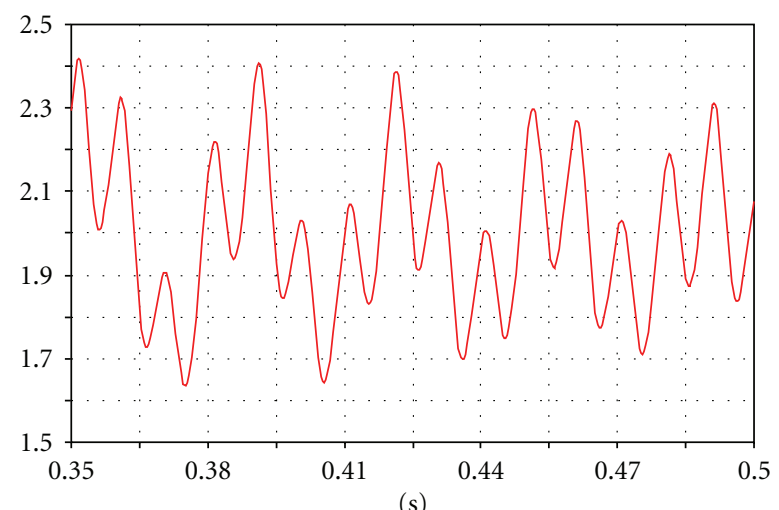

Figure 11: Equivalent conductance at full load for $k_{p}=0.2$ and $k_{i}=3$.

TABLE 4: Most significant current harmonic components when working at the stability limit.

\begin{tabular}{lcc}
\hline Harm. & Amplitude $[\mathrm{A}]$ & $\mathrm{pu}$ \\
\hline 1 & 2038.9 & 1 \\
3 & 110.68 & 0.054 \\
8 & 87.856 & 0.043 \\
10 & 345.03 & 0.169 \\
12 & 76.157 & 0.037 \\
19 & 68.314 & 0.033 \\
21 & 52.113 & 0.025 \\
23 & 20.975 & 0.010 \\
28 & 32.188 & 0.015 \\
\hline
\end{tabular}

switching frequency of the power GTO due to the high rated power of the converter. Therefore, many harmonic components are generated in the input current. The most significant harmonic components are reported in Table 3.

In particular, considerable amplitude is associated to the 10th harmonic order, corresponding to the switching frequency of the converter (17\% of the fundamental component). Moreover, there is the third harmonic component (which has an amplitude that is $2.7 \%$ of the fundamental amplitude) and is caused by the interaction between the 
ripple at $100 \mathrm{~Hz}$ of the DC link voltage and the supply voltage at $50 \mathrm{~Hz}$. The THD is equal to $19.07 \%$.

With respect to the DC link voltage, its behavior at the end of the transient state condition is shown in Figure 7.

The mean value of the voltage is equal to $1800 \mathrm{~V}$, and it is maintained at a constant value equal to the reference value by the PI regulator. Figure 7 shows the clear oscillation at $100 \mathrm{~Hz}$ due to the oscillation of the power absorbed by the singlephase converter.

(B) Loading. The loading case, obtained applying a step load to the system, has been simulated in order to stress the system with high-power variation for stability test. Indeed, the transient behaviour has been analyzed in the case of a load change on the DC side from $50 \mathrm{~A}$ (presuming that $10 \%$ is dedicated to the auxiliary services functioning) to $500 \mathrm{~A}$. This means that a power variation of 3.24 MW (from $360 \mathrm{~kW}$ to $3.6 \mathrm{MW}$ ) has been simulated. Also in this case, the parameters $k_{p}=0.1$ and $k_{i}=3$ obtained for the stability condition are employed in the model.

The absorbed current behaviour in the loading transient at $t=0.3 \mathrm{~s}$ (instant at which the initial simulation transient is finished) is shown in Figure 8.

Moreover, this power variation causes a DC link voltage swell equal to $30-35 \mathrm{~V}(1.6 \%)$, as shown in Figure 9 in continues line. In fact, in the transient phase, the unbalance between requested/remised power from the traction motors and absorbed/injected power from the $4 \mathrm{Q}$ converter, caused by the delay of the PI regulator, is compensated by the energy stored in the smoothing capacitors. The speed of the regulator is an important design parameter because it affects the dynamic of the DC voltage during transient events. A lower speed can cause high overvoltages that can damage the electronic components or deep voltage sags that can lead to loss of converter control.

The DC link voltage ripple implies that the measured value is not suitable for the regulator because it will cause the absorption of a high $3^{\circ}$ harmonic current as shown in (13). For this reason, a filter that limits the $100 \mathrm{~Hz}$ component, but is sufficiently fast to follow the DC link voltage transients, is inserted. The filtered input signal of the converter regulator is shown in Figure 9 by the dashed line. In order to guarantee the stability of the system, this filtered signal has to limit the $100 \mathrm{~Hz}$ component before and after the transient phase maintaining, at the same time, a fast response to follow the transient phenomena.

(C) Converters Regulators Working at the Stability Limit. This last case has been simulated in order to verify the efficacy of the choice of the regulator parameters. For this reason, different values of $k_{p}$ and $k_{i}$ have been inserted in the model. In particular, the following examples will demonstrate the results obtained using the same values obtained previously for the limit of the stability condition: $k_{p}=0.2$ and $k_{i}=3$.

The DC link current and the conductance at full load are demonstrated in Figures 10 and 11. For both of these behaviours it is possible to note a clear increase of an irregular ripple due to the control operation at the limit of stability. These results confirm the theoretical analysis carried out.
The equivalent conductance at the limit of stability assumes, at the end of the transient state condition, a mean value equal to the value at the stability condition because it is correlated to the power transfer requested by the loads. However, in this last case, there is a highly irregular oscillation around this mean value that, combined with the input sinusoidal voltage, causes the absorption of important current harmonic components (Table 4).

\section{Conclusions}

In this paper the influence of controller parameters on the quality of the absorbed current has been investigated. Moreover the application of a current control method to four-quadrant $(4 \mathrm{Q})$ input stage converters employed in traction systems has been proposed. In particular, it has been chosen the Smart Modulation technique with good results, that has been originally developed for low power high switching frequency converters. The changing of modulation technique, from the traditional PWM to the innovative Smart Modulation, has requested a new and deep stability analysis due to the changing of the mathematical equations that describe the whole system. This analysis has also led to a methodology for setting the regulator parameters.

In order to validate the theoretical analysis above described, a circuital model of real cases referred to European High Speed Trains has been implemented in EMTP environment. The outputs of the numerous simulations carried out confirm the results of the theoretical analysis and furnish important indications for the performance of the train and the observance of the European Technical Specification for the Interoperability [1]. In particular, they show how the oscillations in the control loop can cause great current harmonic absorption that can interfere with the track signalling system.

\section{Appendix}

The Smart Modulation (SM) technique is a predictive control that imposes the switching frequency independently from operating conditions. In addition, the SM allows interlacing of switching instants in case of more converters connected in parallel. Referring to Figures 2 and 3, this algorithm considers the reference current $I_{\text {ref }}$ and the inductor flux error $\lambda=L \cdot\left(i-i_{\text {ref }}\right)$ to calculate the commutation instants. The expression that defines the ideal voltage $V_{2}$ at the $4 \mathrm{Q}$ AC terminals when the reference current is absorbed is

$$
v_{2}=e_{2}+L \cdot \frac{d i_{\text {ref }}}{d t}+R \cdot i_{\text {ref }} .
$$

Considering that the trajectories of the real current are linear in a switching period $T$, it is possible to calculate the commutation instants as the intersection of such trajectories:

$$
\begin{aligned}
& \Delta t_{-}=\frac{\left(v_{2}+v_{d}\right)(T / 2)-\lambda}{v_{d}}, \\
& \Delta t_{+}=\frac{\left(v_{d}-v_{2}\right)(T / 2)+\lambda}{v_{d}} .
\end{aligned}
$$


A more detailed description of this algorithm can be found in $[10,11]$.

\section{References}

[1] European Technical Specification for Interoperability (TSI) of the Railway Lines, 2008.

[2] M. Brenna, F. Foiadelli, G. C. Lazaroiu, and D. Zaninelli, "Four quadrant converter analysis for high speed trains," in Proceedings of the 12th International Conference on Harmonics and Quality of Power, Cascais, Portugal, 2006.

[3] S. Ostlund, "Reduction of transformer rated power and line current harmonics in a primary switched converter system for traction applications," in Proceedings of the 5th European Conference on Power Electronics and Applications (EPE '93), vol. 7, pp. 112-119, Brighton, UK, september 1993.

[4] S. Burdett, J. Allan, B. Mellitt, and J. Taufiq, "A power factor and harmonic comparison of AC railway power electronic traction converter circuits," in Proceedings of the 5th European Conference on Power Electronics and Applications (EPE'93), pp. 235-240, Brighton, UK, 1993.

[5] W. Runge, "Control of line harmonics due to four-quadrantconverter in AC tractive stock by means of filter and transformer," in Proceedings of the 7th European Conference on Power Electronics and Applications (EPE '97), pp. 3.459-3.464, Trondheim, Norway, 1997.

[6] G. W. Chang, H. W. Lin, and S. K. Chen, "Modeling characteristics of harmonic currents generated by high-speed railway traction drive converters," IEEE Transactions on Power Delivery, vol. 19, no. 2, pp. 766-773, 2004.

[7] C. Bachle, H. P. Bauer, and T. Seger, "Requirements on the control of a three-level four quadrant power converter in a traction application," in Proceedings of the 3rd European Conference on Power Electronics and Applications (EPE '89), vol. 2, pp. 577-582, Aachen, Germany, 1989.

[8] J. Carter, C. J. Goodman, and H. Zelaya, "Analysis of the singlephase four-quadrant PWM converter resulting in steady-state and small-signal dynamic models," IEE Proceedings Electric Power Applications, vol. 144, no. 4, pp. 241-247, 1997.

[9] D. Casadei, J. Clare, L. Empringham et al., "Large-signal model for the stability analysis of matrix converters," IEEE Transactions on Industrial Electronics, vol. 54, no. 2, pp. 939950, 2007.

[10] M. Brenna, G. C. Lazaroiu, G. Superti-Furga, and E. Tironi, "Bidirectional front end converter for DG with disturbance insensitivity and islanding-detection capability," IEEE Transactions on Power Delivery, vol. 23, no. 2, pp. 907-914, 2008.

[11] M. S. Carmeli, F. Castelli Dezza, and G. Superti Furga, "Smart modulation: a new approach to power converter control," in Proceedings of the 9th European Conference on Power Electronics and Applications (EPE'01), Graz, Austria, August 2001. 

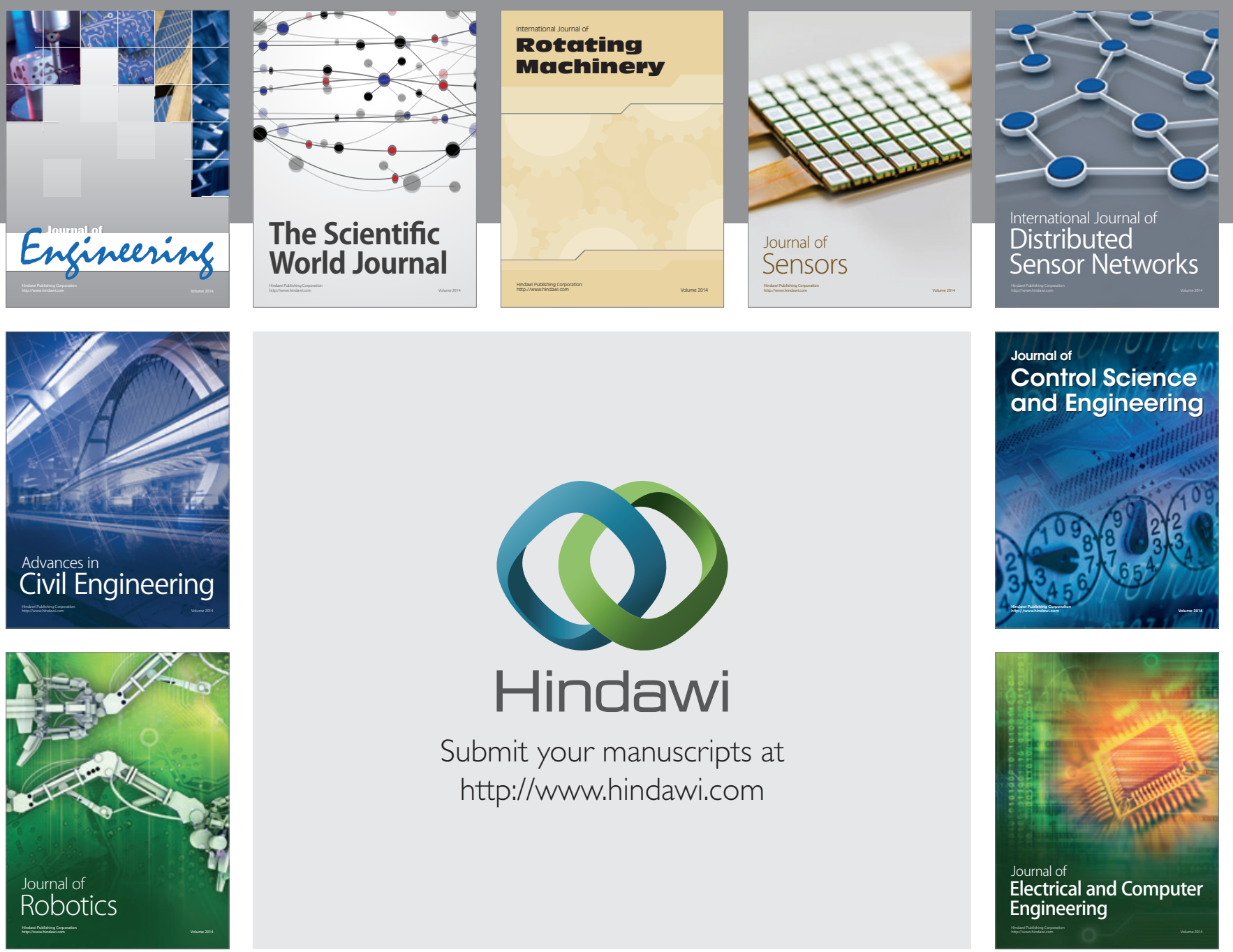

Submit your manuscripts at

http://www.hindawi.com
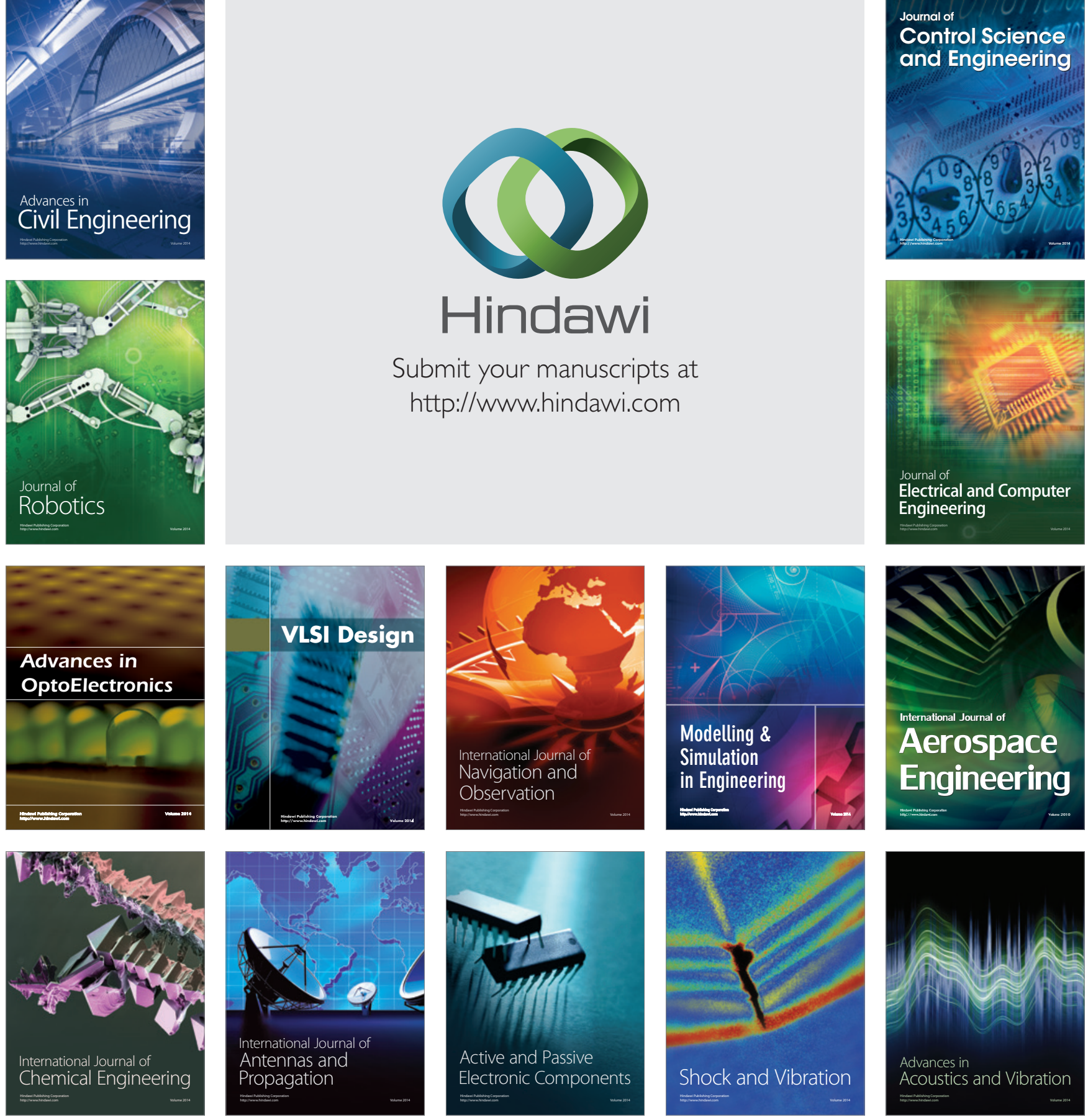